\title{
Effects of Material Characteristics on Flame Spreading ${ }^{1}$
}

\author{
TAKASHI KASHIWAGI, ATSUMI OMORI ${ }^{2}$, and JAMES E. BROWN \\ Center for Fire Research \\ National Bureau of Standards \\ Gaithersburg, Maryland 20899, USA
}

\section{ABSTRACT}

Effects of initial molecular weight and thermal stability of polymer samples on horizontal flame spreading behavior and spread rate were studied by comparing results between two polystyrene, PS, samples with different initial moleculax weights and between two poly(methyl methacrylate), PMMA, samples with different thermal stability and initial molecular weights. The flame spread rate of the higher molecular weight PS sample was about $25 \%$ larger than that for the low molecular weight pS sample and the flame spread rate of the higher molecular weight PMMA sample was about four times larger than that for the low molecular weight sample. The sample with high initial molecular weight does not form molten polymer near the flame front and the flame spreads steadily. However, the sample with low initial molecular weight forms molten polymer and the opposed slow fluid motion of molten polymer along the inclined vaporizing surface against the traveling flame significantly affects flame spreading behavior and its rate.

\section{INTRODUCTION}

It is of great interest to understand the relationship between a polymer's structure and its flammability characteristics. One of the important flammability characteristics is flame spreading which significantly affects fire growth. Although there are extensive studies for the effects of wind velocities, gas phase oxygen concentration, preheating by external thermal radiation, and gravity on the flame spreading mechanism[1-7], there is little understanding of the effects of material characteristics on flame spreading. In effect, most previous detailed studies used PMMA samples with high initial molecular weight over which flame spreads relatively cleanly, avoiding the complex melting process.

In this study, initial molecular weight and thermal stability of the sample were selected as two material characteristics whose effects on flame spreading were to be investigated. Since melt viscosities of molten polymers depend strongly on their molecular weight[8], the effects of initial molecular weight on flame spreading would show whether the melting characteristics of the sample affect flame spread or not. It is also

1 Contribution from the National Bureau of Standards, not subject to copyright.

2 Guest scientist from National Research Institute for Pollution and Resources, Tsukuba, Japan. 
important to find how much the thermal stability of the sample affects flame spreading without significantly modifying the gas phase chemical reaction process. Two types of polymers, PMMA and PS, were selected because the thermal degradation of PMMA is mainly via the depropagation reaction $[9,10]$, and the thermal degradation of PS is mainly via the intermolecular- and intramolecular-transfer reaction $[11,12]$. Thus, it i expected that the thermal stability of PMMA is sensitive to initial molecular weight due to a change in the number of weak linkages in the polymer chains $[9,10]$, but the thermal stability of PS is not sensitive $t$ initial molecular weight. Flame spreading experiments using two PS samples with two different initial molecular weights should indicate onl the effects of initial molecular weight of the sample on flame spreading Flame spreading experiments using two PMMA samples with two different initial molecular weights should indicate the combined effects of initia molecular weight and thermal stability of the sample on flame spreading.

This paper describes the difference in thermal stability between the two different PMMA samples and also between the two different PS samples determined by thermogravimetric analysis and the difference in flame spreading phenomena and spread rate between the samples (mainly in the horizontal flame spreading mode).

\section{EXPERIMENTAL DESCRIPTION}

\subsection{Material}

The two PMMA samples were ELVACITE $2009^{3}$ and 2041 (E.I. Dupont de Nemours \& Co.) and the two PS samples were STYRON 6065 and 6069 (Dow Chemical $\mathrm{Co}$.$) . The original form of these samples was a powder and the$ initial number average molecular weights of these samples measured by ge permeation chromatography were 47,000 and 200,000 for ELVACITE 2009 and 2041, respectively, and 64,000 and 120,000 for STYRON 6065 and 6069, respectively. Therefore, in this paper ELVACITE 2009 is referred to as low MW PMMA sample and 2041 as high MW PMMA sample. Similarly, STYRON 6065 is referred as low MW PS sample and 6069 as high MW PS sample. The samples were cast to make a plate by B.F. Goodrich Co.. The sample specimens for the flame spreading study were about $10 \mathrm{~cm}$ wide $\mathrm{x} 36 \mathrm{~cm} 10$ $\mathrm{x} 1.3 \mathrm{~cm}$ thick.

\subsection{Thermogravimetry}

Weight loss from the sample was measured using a Mettler Thermoanalyz TA 2000. The sample was a powder and a typical sample weight was about $5 \mathrm{mg}$. The reproducibility of weight loss rate was generally excellent an the temperature at the peak weight loss rate could be reproduced to with $1{ }^{\circ} \mathrm{C}$.

\subsection{Flame Spreading}

A horizontally mounted sample on a Marinite plate $(1.3 \mathrm{~cm}$ thick) was placed in the rectangular opening of another Marinite plate close to the sample size and the sample surface was flush with the Marinite surface. The small space between the sample wall and the opening of the Marinite plate was filled with fiber glass insulation material. All horizontal flame spread experiments were conducted in a large Marinite enclosure without a floor (floor covering test apparatus of about $100 \mathrm{~cm}$ high $x 13$ $\mathrm{cm}$ long $\mathrm{x} 40 \mathrm{~cm}$ wide) and combustion products were exhausted through the opening at the top. This enclosure significantly reduced room drafts bu

3 In order to adequately describe materials and equipment it is occasionally necessary to identify commercial products by manufacturer's name. In no instance does such identification imply endorsement by the National Bureau of Standards nor does it imply that the particular produ is necessarily the best available for that purpose. 
induced slight heat feedback from the warm wall of the enclosure during the experiment. Flame spread was initiated by igniting across the $2 \mathrm{~cm}$ wide sample surface at the one end of the sample with a small gas torch. A $25 \mu \mathrm{m}$ wire diameter chromelalume 1

thermocouple spread across the surface of the sample parallel to the flame front, with the junction near the center of the sample, was used to measure the local surface temperature history. To assure good contact between the thermocouple and the sample surface, the thermocouple was heated electrically and simultaneously pressed to the surface prior to a test.

\section{RESULTS}

\subsection{Thermal stability}

Derivative thermogravimetry (DTG), i.e., weight loss rate vs temperature, of the four samples was measured at a heating rate of 5 ${ }^{\circ} \mathrm{C} / \mathrm{min}$. The DTG results were obtained by numerically taking the time derivative, $\left(d\left(W / w_{0}\right) / d t\right)$, of the ratio of the sample weight, $W$, to the initial sample weight, $W_{0}$. Results for the PS samples are shown in Fig.1. Although the results of the cast plate of PS samples are not shown in Fig. I in order to avoid crowding the figure, the casting process hardly modified the thermal stability of the PS samples. Since thermal oxidative degradation and thermal degradation might occur near the surface[13], degradation characteristics were determined both in nitrogen and in air, and the results are shown in Fig.1. The results show that there is only one sharp peak in nitrogen and one global peak consisting of two overlapped peaks in air. The PS samples degrade at much lower temperatures in air than in nitrogen probably due to the formation of hydroperoxides and the degradation of hydroperoxides at low temperatures[14]. The important result obtained from these figures is that there are no significant differences in thermal stability between the two PS samples, as we expected.

Similar results for the two PMMA samples are shown in Fig.2. There are three peaks for the high MW sample degrading in nitrogen. Since this sample was polymerized by a free radical method, it is expected that the first peak at low temperature (around $170^{\circ} \mathrm{C}$ ) is caused by scission at the weak linkage of the head-to-head structure[10,15], the second peak at moderate temperature (around $280^{\circ} \mathrm{G}$ ) is due to radical initiation at the unsaturated chain ends $[9,10,15]$, and the third peak at high temperature (at around $370^{\circ} \mathrm{C}$ ) is due to random scission at the main chain Iinkages. 
If the polymerization method for the low MW sample were exactly the same as that for the high MW sample, peak heights for the first two peaks for the low MW sample should be higher than those for the high MW sample due to the increase in the number of weak linkages for a low initial molecular weight PMMA sample[10]. However, the results of the low MW sample show one large third peak with extremely little weight losses at the positons of the first two peaks. This suggests that the low MW sample was polymerized with a chain transfer agent to avoid the formation of the weak linkages in polymer chains during its polymerization process $^{4}$ and consequently the initial molecular weight of the low MW sample became much smaller than that of sample $B[10]$. Results of the samples degrading in air show an increase in thermal stability at the low temperature range (the disappearance of the first peak for the high MW sample) and significant reduction in thermal stability at the high temperature range (shift of 1 third peak toward lower temperature). This is consistent with our previous study of oxygen effects on PMMA degradation[10], such as trapp: of polymer radicals initiated by chain scission at the head-to-head linkages by oxygen and also the formation of hydroperoxides and their thermal degradation.

\subsection{Flame spreading}

\subsubsection{PS Samples (Effects of Melting)}

The approximate expression of polymer melt viscosity, $\eta$, is $\eta=\mathrm{cm}^{3} .4$ where $M$ is molecular weight of the sample and $c$ is a constant dependent the polymer. Using this expression, the melt viscosity of the high MW ? sample is about one order of magnitude higher than that of the low MW P: sample.

Flame spread over the surface of the high MW PS sample with a reasonably steady rate as shown in Fig. 3 except for the initial flame growth period. In this study, time zero was defined when the traveling flame front reached $4 \mathrm{~cm}$ from the end of the sample. A schematic illustration of the flame over the high MW sample is shown in Fig. $4 \mathrm{a}$. burning surface appeared to be watery with many medium size bubbles (abs $1 \mathrm{~mm}$ diameter). The flame was sooty and quite bright; the traveling $\mathrm{fl}$; front was well defined and its color was blue. A small bulge due to

4 It was confirmed by E.I. Dupont de Nemours \& Co. that sample A wa polymerized using a chain transfer agent. 
melting of the sample was observed in front of the flame. The distance between the flame front and the sample burnout front (end of flame) was 10-12 cm after the flame spread rate reached near steady state. The angle of the inclined sample surface covered by flame was roughly $7^{\circ}$.

Flame spread behavior over the surface of the low MW PS sample was quite transient and different from the high MW PS sample. As shown in Fig. 3 , initially the flame spread very slowly. A schematic illustration of the flame spread phenomenon during this period is shown in Fig. $4 \mathrm{~b}$. Since the flame spread forward so slowly while the sample surface regressed, a steep wall of molten polymer appeared in front of the flame. There was no visible blue flame front. When a small particle was put on the top of the wall, a slow downward movement of the particle toward the bottom of the wall was observed, which indicates the existence of a slow fluid movement of the molten polymer. It appeared that

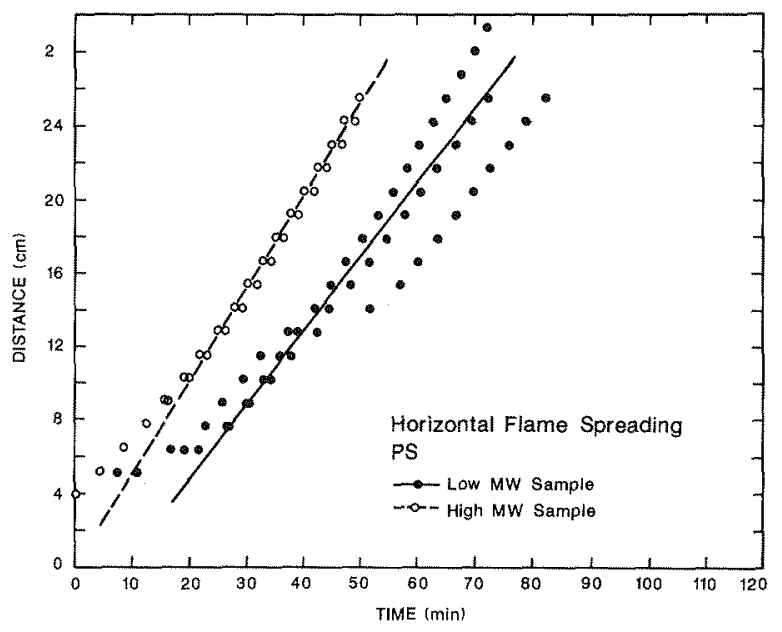

FIGURE 3. Plot of flame position with time for PS samples; average flame spread rate for the low MW sample $6.7 \pm 1.3 \times 10^{-3} \mathrm{~cm} / \mathrm{s}$ and $8.5 \pm 0.4 \times 10^{-3} \mathrm{~cm} / \mathrm{s}$ for the high MW sample.
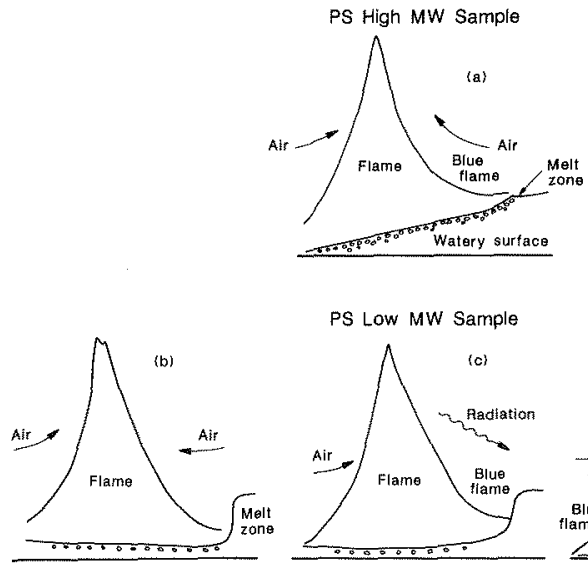

PS LoW MW Sample
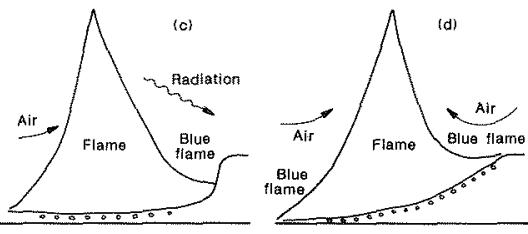

Figure 4. A schematic illustration of flame spread behavior for the PS Samples. (a) Over the high MW sample, (b) Over the low MW sample with the wall, (c) over the low MW sample with the flame climbing the wall, and (d) Over the low MW Sample with near steady state spreading.

this opposing slow

fluid movement against flame spreading caused the slowdown of flame spread and consequently the formation of the steep wall. After the flame spread 
slowly to about $8 \mathrm{~cm}$ from the beginning of the sample with the conditior illustrated in Fig. 4b, the flame started to climb the steep wall as described in Fig.4c. At this point, the flame became tall (roughly 17 c high) and the thermal radiation feedback from the flame to the surface ( the steep wall became large enough to evolve sufficient amount of degradation products for the flame to be able to climb the steep wall. The color of the flame front again became blue. The flame continued climbing to the top of the wall. When the flame front reached about 14 $\mathrm{cm}$, the beginning portion of the sample burned out. Then, the size of $t$ flame became smaller and this slowed down flame spread due to a decreast in thermal radiation feedback from the flame to the sample surface. The width of the flame became $7.8 \mathrm{~cm}$ when flame spread at the near steady state condition. Flame spread as described in Fig.4d with the blue flan at its front and the burning sample surface was more sharply inclined th that of the high MW PS sample.

The average flame spread rate, excluding the beginning part of the flame spread, was estimated for each sample from the results shown in Fig. 3. It was $6.7 \pm 1,3 \times 10^{-3} \mathrm{~cm} / \mathrm{s}$ for the low MW PS sample and $8.5 \pm 0.4 \times 1 \mathrm{C}$ $\mathrm{cm} / \mathrm{s}$ for the high MW PS sample. Therefore, the flame spread roughly 25 ? faster over the high MW PS sample than ove: the low MW PS sample. The surface temperature distribution near the flame front was obtained from the measured time history of surface temperature multiplied by the measured local flame spread rate. The comparison of the surface temperature distributions between the two PS samples is shown in Fig. 5 . Although there is no significant difference between them, the surface temperature distribution for the high MW sample is slightly steeper near the flame front

than that for the

low MW sample.

Both results show strong

melting of the sample by a sharp kink in

temperature distribution at about $0.2 \mathrm{~cm}$ before the vaporization front. Another interesting fact is that the surface temperature even at $1.5 \mathrm{~cm}$ ahead of the vaporization front is as high as about $110^{\circ} \mathrm{C}$. As discussed later, this temperature is much higher (at least $40^{\circ} \mathrm{C}$ ) than

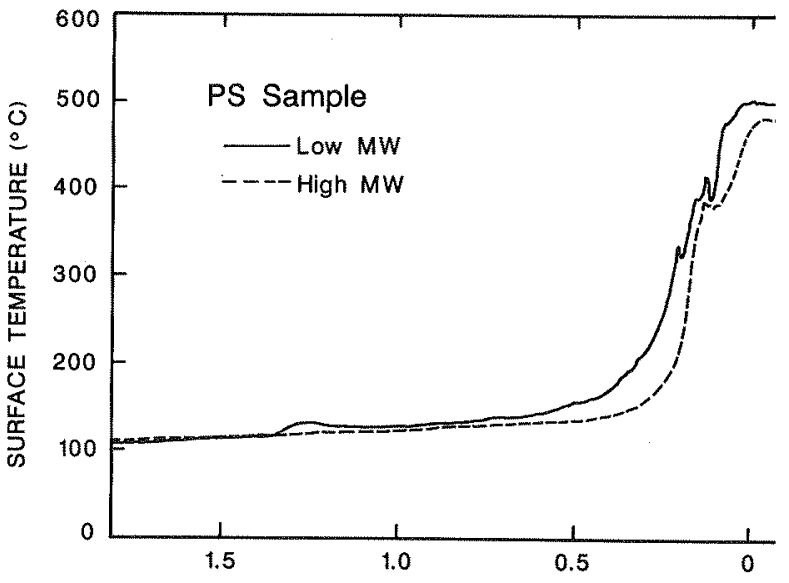

DISTANCE AHEAD OF VAPORIZATION FRONT $(\mathrm{cm})$

Figure 5. Comparison of surface temperature distribution near the flame front between the two samples.

that for the PMMA

samples, which

indicates the importance of radiation feedback from flame to the sample surface ahead of the flame front for PS samples. 


\subsubsection{PMMA Samples (Effects of Melting and Thermal Stability)}

It is estimated that the melt viscosity of the high MW sample is about two orders of magnitude larger than that of the low MW sample using the same relationship between melt viscosity and molecular weight used for the above PS samples. Therefore, the difference in melt viscosity between the two PMMA samples is about one order of magnitude larger than that for the above PS samples. Furthermore, there is a difference in thermal stability between the two PMMA samples. Thus, the difference in flame spreading between the two PMMA samples is caused by the combined effects of melting and thermal stability characteristics.

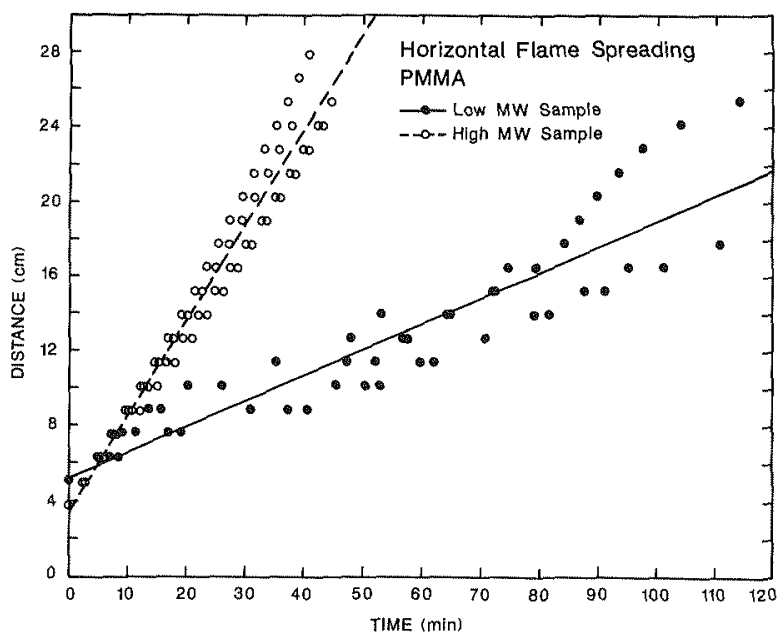

FIGURE 6. Plot of flame position with time for PMMA samples; average flame spread rate for the low MW sample was $2.3 \pm 1.0 \times 10^{-3} \mathrm{~cm} / \mathrm{s}$ and $8.5 \pm 0.5 \times 10^{-3}$ $\mathrm{cm} / \mathrm{s}$ for the high MW sample.

The relationship between the location of the flame front and time shown in Fig. 6 indicates relatively steady flame spread for the high MW sample and quite unsteady spread for the low MW sample. Flame spread over the high MW sample with a clear blue flame front which appeared to be extended slightly ahead of the vaporization front. As shown in Fig.7a, there were many small bubbles near the burning surface but its surface was not as watery as that for the PS samples. The width of burning surface (between the flame front and the burnout front) was roughly $15 \mathrm{~cm}$ when steady flame spreading was attained. The inclination angle of the burning surface was very gentle and overall flame spread behavior was very similar to that over Plexiglas $G$ which was often used as a sample in previous studies.

Flame spread over the low MW sample was quite complex, similar to flame spread over the low MW PS sample. When flame spread to about the $8 \mathrm{~cm}$ location, flame spread very slowly. The molten surface near the vaporization front appeared to be watery, causing the opposed fluid motion of molten PMMA by the inclination of the vaporizing sample surface. The energy feedback from the flame to the surface ahead of the vaporization front appeared to be insufficient to degrade the thermally stable low MW sample ahead of the vaporization front. Therefore, the regression rate normal to the surface was slightly larger than the flame spread rate, and the wall described in Fig. $7 \mathrm{~b}$ was formed. Once the wall was formed, the blue flame front was absent and the flame almost stopped spreading. When the flame reached around the $10 \mathrm{~cm}$ location, the flame behavior was still the same as described in Fig. 7b. Then, the burnout of the downstream part of the sample occurred and the center of the flame moved forward due to narrowing of the width of the flame by the burnout (from about $10 \mathrm{~cm}$ to 
$5 \mathrm{~cm}$ ). Under

this condition, air was

entrained mainly

from the back

side of the

flame instead of

the normal case

in which air was

entrained from

both sides

(front and back)

of the flame.

It appeared that

the wall acted

as an obstacle

to air

entrainment from

the front side

of the small

flame. This

caused the flame

to lean forward

and to move back

and forth as

described in

Fig. 7c.

Although there

was no visible

blue flame front, energy feedback from the flame to the wall was

temporarily enhanced when the flame leaned forward. The wall was rapid1 smoothed by the enhanced feedback and the flame climbed partially over $t$ step. However, there was no blue flame front as shown in Fig.7d. The flame behavior was more or less similar to flame spread aided by a force wind or upward flame spread. Entrained air from the back side of flame acted as the forced wind and the flame continued to spread in this mode. The flame spread rate appeared to be sensitive to the aerodynamics of th air entrainment which was also sensitive to the shape of the burning surface contour. Therefore, there was some scatter in the flame spread rate over the low MW sample. The width of the burning surface was about 5-6 $\mathrm{cm}$, the narrowest in this study, and the inclination angle of the burning surface was very steep. The slow flow of molten polymer along $t$ inclined surface, similar to the low MW PS sample, was also observed for the low MW PMMA sample. The average flame spread rate was calculated fr the results shown in Fig. 6 . It is $2.3 \pm 1.0 \times 10^{-3} \mathrm{~cm} / \mathrm{s}$ for the low MW PMMA sample and $8.5 \pm 0.5 \times 10^{-3} \mathrm{~cm} / \mathrm{s}$ for the high MW sample. Therefore, the flan spread roughly four times faster over the high MW sample than over the 1 MW sample. The difference in the above-discussed flame front behavior between the two PMMA samples is also demonstrated by the comparison of surface temperature distribution between them, as shown in Fig. 8 . The temperature distribution for the high MW sample shows the sharp increase within about $2 \mathrm{~mm}$ ahead of the vaporization front indicating a sharp, we defined flame front. However, the temperature distribution for the low sample shows the much more gradual increase, with fluctuations presumabl caused by the unstable flame movement. The surface temperature at $1.5 \mathrm{c}$ ahead of the vaporization front was about $60-70^{\circ} \mathrm{C}$ for the PMMA samples compared with about $110^{\circ} \mathrm{C}$ for the PS samples. This indicates the importance of radiation feedback from the flame to the unburned sample surface for flame spread over the PS samples. 


\section{DISCUSSION}

The above results show the importance of initial molecular weight of the sample for horizontal flame spreading due to its effect on the melt viscosity of the molten polymer near the flame front. A lower initial molecular weight results in a lower melt viscosity of the molten polymer which induces a slow fluid movement against the spreading flame. The opposed flow of molten polymer is caused by the slight inclination of the sample surface neax the vaporization front. If there is a PS sample with lower initial molecular weight than the PS samples used in this study, it is expected that the horizontal flame spread rate should be lower that those values measured in this study. Since the differences between the two PMMA samples are initial molecular weight and thermal stability, it is not clear how much each effect contributed to the fact that the flame spread rate for the high MW sample was four times larger than that for the low MW sample. However, since there is a large difference in piloted radiative ignition delay time between the two PMMA samples[16] under same external radiant fluxes, and also since it was difficult to ignite the low $\mathrm{MW}$ sample by a torch for the start of the flame spreading experiment, the thermal stability of the material should have significant effects on flame spreading. However, the quantitative effects of the difference in the DTG curves shown in Fig. 2 on flame spreading rate can not be clearly determined because there is no unique vaporization temperature (most models need this value to estimate flame spread rate) in the DTG curves.

other important parameters are the sample thickness and the inclination of the burning sample surface. The sample thickness has been considered to be important only for the energy transfer through the sample in previous studies as a thermally thin or thermally thick sample. However, this study indicates that it has another aspect, which is the width of the burning sample surface between the flame front and the burnout front. This determines the flame size which determines the air entrainment characteristic induced by buoyancy and also the radiation feedback rate from the flame to the sample surface ahead of the vaporization front. This is especially important for horizontal flame spreading. It appears that slower flame spreading tends to form the steeper inclination of the burning sample surface behind the flame front. However, at present, the majority of theoretical models for flame spread assume that the surface remains flat except Altenkirch et al[17]. The effects of the inclination of the burning surface on the flame spreading mechanism should be more investigated. 
It is anticipated that the effects of melting of polymer on downward flame spreading should be more significant than the horizontal flame spreading. A limited number of downward flame spreading experiments wer conducted to obtain preliminary information. Downward flame spread over the high MW PMMA sample did not show any dripping and the flame spread steadily. However, the flame over the high MW PS sample spread with a much-enhanced rate compared with the rate for horizontal flame spread, d to streaking of small molten polymer balls similar to downward flame spread behavior observed with Plexiglas G[18]. The flame over the low $M$ PS sample and the 10w MW PMMA sample was self-extinguished during downwa flame spreading due to the following two causes: (1) small burning molte polymer balls were formed at the tips of the saw tooth shape vaporizatio front and these streaked rapidly downward to the cold sample where they self-extinguished due to heat loss to the cold surface, and (2) these streaking molten polymer balls carried away thermal energy from the base part of the saw tooth shape vaporization front and subsequently the flam extinguished. Therefore, the effects of polymer melting on downward fla spreading are complex in that they enhance flame spreading when the melt viscosity of the molten polymer is relatively high but extinguish the flame when the melt viscosity becomes too low.

\section{CONCLUSIONS}

(1) The initial molecular weight of the sample has a significant effect horizontal flame spreading behavior and its rate. The sample with high initial molecular weight does not form molten polymer near the flame fro and the flame spreads steadily. However, the sample with low initial molecular weight forms molten polymer and the opposed slow fluid motion molten polymer along the inclined vaporizing surface against the traveli flame significantly affects flame spreading behavior and its rate.

(2) Flame spread rates over the two PMMA samples differ by almost four times due to the combined effects of thermal stability and initial molecular weight. This clearly indicates that material characteristics significantly affect flame spread behavior and its rate. (3) The sample thickness is an important parameter not only in the conventional energy loss aspect through the sample but also in determining the flame size du to the sample burnout.

\section{ACKNOWLEDGMENT}

This study is partially supported by B.F.Goodrich Co.. The authors would like to thank for Dr. Marcelo Hirschler and Mr. Andrew Pazur of B.F.Goodrich Co. for their purchase of the polymer samples used in this study and casting them to make plates and Mrs. Robin Breese and Mr. Chri Tate for their assistance in conducting the experiments.

\section{REFERENCES}

1. De Ris, J., Twelfth Symposium (International) on Combustion, The Combustion Institute, Pittsburgh, p. 241, 1969.

2. Altenkirch, R. A., Eichhorn, R., and Rizvi, A.R., Combust. Sci. Tect 32: $49,1983$.

3. Fernandez-Pello, A. C., and Williams, F. A., Fifteenth Symposium (International) on Combustion, The Combustion Institute, Pittsburgh, p. 217,1975 .

4. Hirano, T., Koshida, T., and Akita, K., Bulletin of Japanese Association of Fire Science and Engineering, 27: 33, 1977.

5. Fernandez-Pello, A. G. and Santoro, R. J., Seventeenth Symposium 
(International) on Combustion, The Combustion Institute, Pfttsburgh, p. $1201,1978$.

6. Ray, S. R. and Glassman, I., Combust. Sci. Tech., 32: 33, 1983.

7. Quintiere, J., Harkleroad, M., and Walton, D., Combust. Sci. Tech., 32: 67,1983 .

8. Matsuoka, S. and Kwei, T. K., "Physical Behavior of Macromolecules" in Macromolecules, ed. Bovey, F. A. and Winslow, F. H., F. 346, Academic Press, New York, 1979.

9. Grassie, N. and Melville, H. W., Proc Royal Soc. London, Ser. A., 199: 14, 1949.

10. Kashiwagi, T., Inaba, A., Brown, J. E., Hatada, K., Kitayama, T., and Masuda, E. Macromolecules, 19: 2160, 1986.

11. Wall, L. A., Straus, S., Flynn, J. H., McIntyre, D., and Simha, R., J. Phy. Chem., 70: 53, 1970.

12. Guyot, A., Polymer Degrad. Stability, 15: 219, 1986.

13. Jakes, K. A., and Drews, M. J., J. Polymer Sci., Polymer Chemist. Ed., 19: 1921,1981

14. Kamiya, Y. and Niki, E., "Oxidative Degradation," in Aspects of Degradation and Stabilization of Polymers, ed. Jellinek, H. H. G., Chapt. 3, Elsevier, Amsterdam, 1978.

15. Solomon, D.H., J. Macromol. Sci. Chem., Al7(2): 337, 1982.

16. Kashiwagi, T., and Omori, A., to be published.

17. Altenkirch, R. A., Rezayat, M., Eichhorn, R., and Rizzo, F. J., I. Heat Transf., 104: 734, 1982.

18. Kashiwagi, T., Inaba, A., and Brown, J. E., Fire Safety Science, Proceedings of First International Symposium, Hemisphere, p. 483, 1986. 
